\title{
Association of recurrent pregnancy loss with chromosomal abnormalities and hereditary thrombophilias
}

\author{
*Ocak Z11, Özlü T², Ozyurt O
}

1. Department of Medical Genetics, Abant Izzet Baysal University Medical School, Bolu, Turkey

2. Department of Obstetrics and Gynecology, Abant Izzet Baysal University Medical School, Bolu, Turkey

3. Department of Obstetrics and Gynecology, Suleymaniye Maternity Hospital for Research and Training, Istanbul, Turkey

\begin{abstract}
Background: Recurrent pregnancy loss (RPL) which is generally known as $>3$ consecutive pregnancy losses before 20 weeks' gestation is seen in $0.5-2 \%$ of women

Objective: To evaluate the association of parental and fetal chromosomal abnormalities with recurrent pregnancy loss in our area and to analyze the frequency of three types of hereditary thrombophilia's; (MTHFR C677T polymorphisms, FV Leiden G1691A mutation and Prothrombin (factor II) G20210A mutation) in these female patients.

Methods: The present case-control retrospective study was performed between February 2007 and December 2011 on 495 couples, who had two or more consecutive pregnancy losses before 20 weeks' gestation. We used conventional cytogenetic analysis and polymerase chain reaction-restriction fragment length polymorphism.

Results: Parental chromosomal abnormality was detected in 28 cases (2.8\% of all cases, $5.7 \%$ of the couples) most of which $(92.9 \%)$ were structural abnormalities. All of the structural abnormalities were balanced chromosomal translocations. Chromosomal analysis performed from the abortion materials detected a major chromosomal abnormality in $31.9 \%$ of the cases. The most frequently observed alteration in the hereditary thrombophilia genes was heterozygote mutation for the MTHFR C677T polymorphisms ( $\mathrm{n}=55)$.

Conclusion: Balanced translocations are the most commonly detected chromosomal abnormalities in couples being evaluated for recurrent pregnancy loss and these patients are the best candidates for offering prenatal genetic diagnosis by the help of which there is a possibility of obtaining a better reproductive outcome.
\end{abstract}

Key words: chromosomal abnormality, recurrent pregnancy loss, thrombophilia African Health Sciences 2013; 13(2): 447 - 452 http://dx.doi.org/10.4314/ahs.v13i2.35

\section{Introduction}

Recurrent pregnancy loss (RPL) which is generally known as $>3$ consecutive pregnancy losses before 20 weeks' gestation is seen in $0.5-2 \%$ of women. ${ }^{1,2}$ It is defined by American Society of Reproductive Medicine (ASRM) as two or more failed pregnancies. ${ }^{3}$ Several etiological factors like endocrinological problems, uterine structural or chromosomal anomalies and prothrombotic conditions can be the cause in some of these cases. However, about $40-60 \%$ of the RPL cases are idiopathic ${ }^{4}$. Chromosomal abnormalities, which are the most common causes of sporadic early

\footnotetext{
*Corresponding author:

Zeynep Ocak

Abant Izzet Baysal Üniversitesi

Tibbi Genetik AD

14280, Gölköy, Bolu, Türkiye

Tel: +903742534656

Fax: +903742534615

E-mail: zeynep_ipek@yahoo.com
}

pregnancy losses, are also reported to be responsible from an important proportion of recurrent losses. ${ }^{5,6}$

It has been a very common practice to blame the hereditary thrombophilias which include Factor V Leiden mutation, Prothrombin G20210A gene mutation, Protein $\mathrm{S}$ deficiency, Protein $\mathrm{C}$ deficiency, Antithrombin deficiency, and methylenetetrahydrofolate reductase (MTHFR) mutations in the pathogenesis of RPL. There are contradictory studies in the literature that either do or do not support the possible role of hereditary thrombophilias in the etiology of RPL.,11

In the present study we evaluated the association of parental and fetal chromosomal abnormalities with RPL. Also, we analyzed the frequency of three types of hereditary thrombophilia's; (MTHFR C677T polymorphisms, FV Leiden G1691A mutation and Prothrombin G20210A mutation) in female patients. 


\section{Methods}

\section{Subjects}

The present case-control retrospective study was performed between February 2007 and December 2011. The study population consisted of 495 couples, who had two or more consecutive pregnancy losses before 20 weeks' gestation. Age of the female patients at their last pregnancy loss was recorded. Couples in whom the woman's history revealed thromboembolism or systemic disease were excluded from the study. The Ethics Committee at Suleymaniye Maternity Hospital for Research and Training approved the use of the clinical information and the collection of samples for research purposes.

\section{Standard cytogenetic analysis}

In order to reveal the karyotype of the patients, 72hour culturing was performed using peripheral blood lymphocytes induced with phytohaemagglutinin (PHA). Metaphase preparates obtained after culturing were stained using the trypsin Giemsa banding method (GTG). Small tissue specimens obtained from the abortion materials were cultured in three separate flasks. In cases in which mosaic karyotype was identified in the abortion material, maternal tissue contamination was excluded by analysis of the materials with small tandem repeat (STR) markers (16 region). Beginning from the 7th day, flasks were controlled in terms of cell proliferation and contamination, and at around the $13^{\text {th }}$ to $14^{\text {th }}$ days cultures with adequate colonies were harvested. CTG banding was performed by conventional cytogenetic methods in all metaphases obtained from all 3 cultures. In metaphases that were suspicious for polymorphisms, $\mathrm{C}$ banding was also performed. The results of the cytogenetic analysis were examined in 3 groups as: 1) Numerical chromosomal abnormalities 2) Structural chromosomal abnormalities 3) Polymorphisms.

\section{PCR method}

Factor V Leiden, Prothrombin G20210A and MTHFR C677'T gene polymorphisms were analyzed by polymerase chain reaction-restriction fragment length polymorphism (PCR-RFLP). The enzymes used for the mutation analyses of Factor $\mathrm{V}$ Leiden, Prothrombin G20210A and MTHFR C677T were Mnl I, Hind III, Hinf I, respectively. The fragments were visualized by ethidium bromide under UV transilluminator.

\section{Results}

A total of 495 couples were included in the study. Mean age of the female patients was 30.6 years (range: 19-44). Parental chromosome analysis was performed in all of the 495 couples. Among these 990 subjects, a major chromosomal abnormality was detected in 28 cases $(2.8 \%$ of all cases, $5.7 \%$ of the couples) (table 1). 16 (57.1\%) of the abnormalities were in females, and, 12 (42.9) of the abnormalities were in males.

Table 1: Spectrum of major chromosomal abnormalities detected from chromosome analysis of 495 couples performed for the investigation of the etiology of recurrent pregnancy loss.

\begin{tabular}{ll}
\hline Structural (n=26) & Numeric (n=2) \\
\hline 46,XY,t(1;11)(q42.1;q13.4) & 45,X[3]/46,XX[47] \\
46,XY,t(2;3)(p13;q27) & 47,XXX[2]/46,XX[55] \\
46,XX,t(2;4)(p24;q13) & \\
46,XX,t(3;8)(q25;p21),9qh+ \\
46,XX,t(3;6)(p25;q13) \\
46,XY,t(3;20)(p14.1;p13) \\
46,XY,t(4;20)(q22;p11.2) \\
46,XY,t(4;22)(q34;q1) \\
46,XX,t(6;9)(p21;p23) \\
46,XX,t(6;11)(q21;q23.3) \\
46,XX,t(8;14)(p21;q32) \\
46,XX,t(8;15)(q22;q15) \\
46,XY,t(8;18)(q22.3;q21.1) \\
46,XY,t(8;22)(p21;q13.1) \\
46,XX,t(9;16)(q22.1;p11.1) \\
46,XX,t(9;20)(q21;p11.2) \\
46,XX,t(10;16)(q25.1;p12) \\
45,XX,rob(13;13)(q10;q10) \\
45,XY,rob(13;14)(q10;q10),22 \\
pstk+ \\
46,XY,t(13;18)(q21;q22) \\
46,XY,(14;21)(q10;q10) \\
45,XX,rob(14;21) \\
45,XY,inv(9)(p11q13),rob(14; \\
22)(q10;q10) \\
46,XX,rob(15;21)(q10;q10) \\
46,XX,rob(15;21)(q10;q10) \\
45,XX,rob(13;13)(q10;q10) \\
\hline
\end{tabular}

There were no couples in whom a chromosomal abnormality was detected in both the male and the female partner. Of these, $2(7.1 \%)$ were numeric and $26(92.9 \%)$ were structural abnormalities. All of the structural chromosomal abnormalities detected in the parents were balanced translocations. Polymorphisms were detected in 316 (31.9\%) of 
the 990 subjects (table 2). The most commonly detected polymorphism was an increase in the centromeric heterochromatin region of the $1^{\text {st }}$ chromosome.

Table 2: Polymorphic chromosomal variants detected in couples with a history of recurrent pregnancy loss

\begin{tabular}{ll}
\hline Polymorphism & Number of cases \\
\hline 1qh+ & 67 \\
1qh- & 6 \\
9qh+ & 22 \\
Inv 9 & 13 \\
13ps & 4 \\
13pstk & 7 \\
13cenh+ & 3 \\
14ps & 5 \\
14pstk & 2 \\
15ps & 12 \\
15pstk & 8 \\
15cenh+ & 20 \\
16qh+ or 16qh- & 38 \\
21ps & 11 \\
21pstk & 3 \\
22ps & 13 \\
22pstk & 6 \\
Yqh+ or Yqh- & 76 \\
Total & 316 \\
\hline
\end{tabular}

Chromosomal analysis from the abortion material was performed in 250 cases, but cell culture was successful in 135 of these. A major chromosomal abnormality was detected in $43(31.9 \%)$ cases, of which $39(90.7 \%)$ were numeric and $4(9.3 \%)$ were structural (table 3). Polymorphisms were detected in $5(3.7 \%)$ of the 135 cases.

Four hundred ninety five females that were included in the study were tested for three types of hereditary thrombophilia's; MTHFR C677T polymorphisms, FV Leiden G1691A mutation and Prothrombin G20210A mutation. Among these, 414 of the women did not carry any of these mutations while any abnormality which could be at least a heterozygote mutation in any of these genes was detected in $81(16.4 \%)$ cases (table 4$)$. The most frequently observed abnormality in this group was heterozygote mutation for the MTHFR polymorphisms $(\mathrm{n}=55)$.

Table 3: Chromosomal abnormalities detected from chromosome analysis of the abortion materials of 250 cases. (Cell culture was successful in 135 cases, unsuccessful in 115 cases)

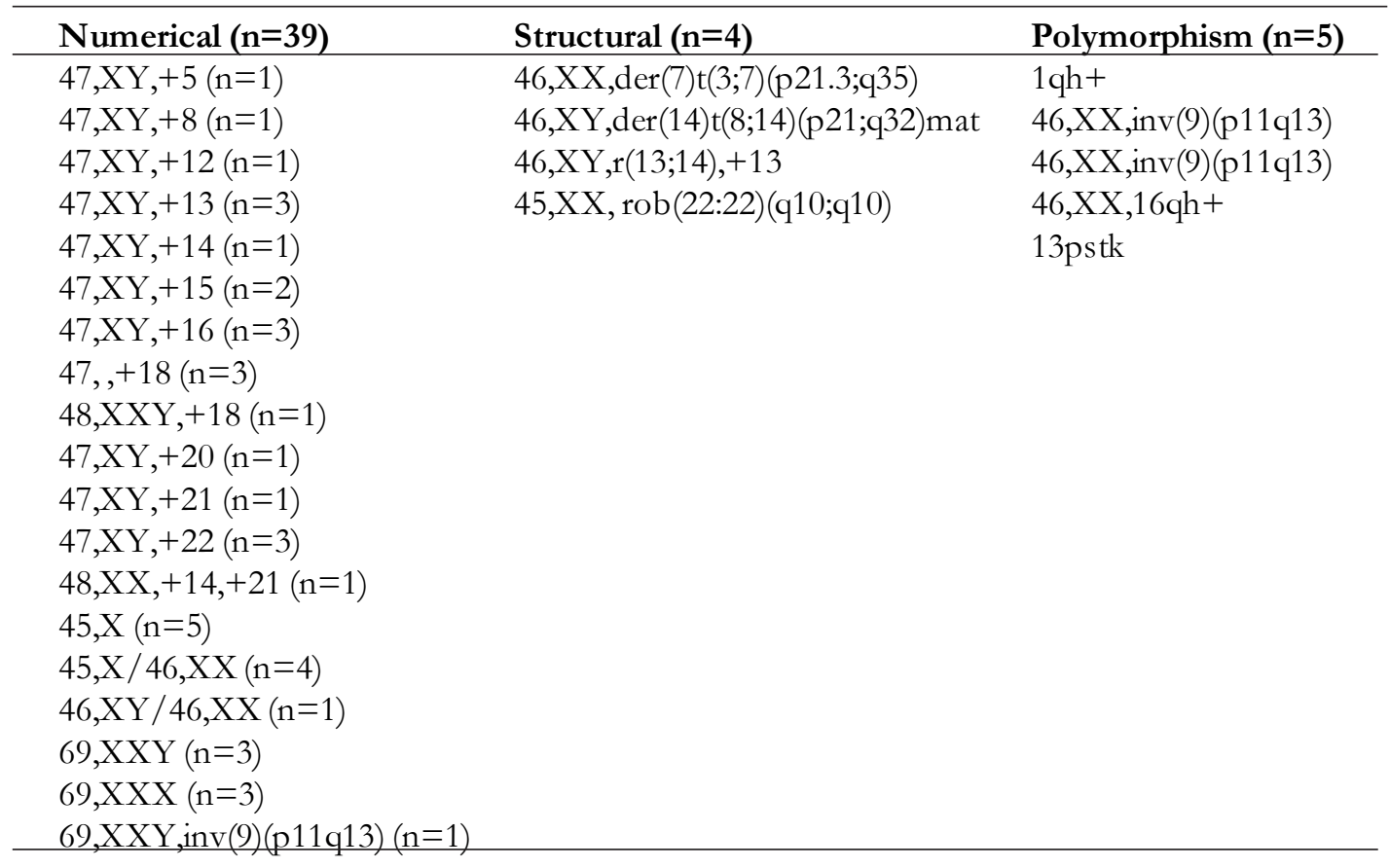


Table 4: Genotypes of the hereditary thrombophilia genes in 81 patients

\begin{tabular}{ll}
\hline Normal & $\mathbf{4 1 4}$ \\
\hline Abnormal & $\mathbf{8 1}$ \\
\hline MTHFR-Homozygous & $2(2,5 \%)$ \\
MTHFR-Heterozygous & $55(67,9 \%)$ \\
FVL Heterozygous/MTHFR- & $9(11,1 \%)$ \\
Homozygous & \\
FVL Heterozygous & $5(6,2)$ \\
FII Heterozygous & $4(4,9 \%)$ \\
$\begin{array}{l}\text { FII Heterozygous / MTHFR } \\
\text { Heterozygous }\end{array}$ & $2(2,5 \%)$ \\
$\begin{array}{l}\text { MTHFR Heterozygous in addition } \\
\text { to a structural chromosomal } \\
\text { abnormality }\end{array}$ & $4(4,9 \%)$ \\
\hline
\end{tabular}

\section{Discussion}

Parental chromosomal abnormalities are detected in about $2-8 \%$ of couples with recurrent miscarriages. ${ }^{12}$ Several types of genetic problems like parental structural chromosomal abnormalities and recurrent aneuploidies may be associated with recurrent miscarriage. Balanced chromosomal rearrangements are found in $2-5 \%$ of these couples, and among these balanced translocations are the most frequent abnormalities ${ }^{13}$. In our study, parental chromosomal abnormality was detected in $2.8 \%$ of all cases and $5.7 \%$ of the couples. As expected, most of these $(92.9 \%)$ were structural abnormalities and all of the structural abnormalities were balanced chromosomal translocations. As we know, these patients are the best candidates for offering prenatal genetic diagnosis (PGD) by the help of which better reproductive outcomes can be obtained ${ }^{14}$ although the data about latter aspect of PGD is still insufficient. ${ }^{15}$ PGD, which has some technical limitations currently ${ }^{16}$ is difficult and it requires in vitro fertilization with or without intracytoplasmic sperm injection which is expensive and may not be convenient in all patients.

Chromosome analysis from the abortion material helps us to detect whether any type of chromosomal abnormality in the fetus is the reason of the abortion. We know from the previous studies that chromosomal abnormalities are responsible from about half of early miscarriages. For example Zhang et al. investigated chorionic villi of 252 cases of missed abortion and detected chromosomal abnormality in $58.09 \%$ of the cases (81 were trisomy, 29 were monosomy $\mathrm{X}$ and 17 were polyploidy). ${ }^{17}$ Similarly, Kwinecka-Dmitriew et al. studied the incidence of chromosomal abnormalities in abortions and detected chromosomal abnormalities in $37.5 \%(45 / 120)$ of the cases (trisomy, triploidy and monosomy $\mathrm{X}$ in decreasing frequency). ${ }^{18} \mathrm{The}$ incidence was $42.4 \%(25 / 59)$ in the first miscarriages and $32.8 \%(20 / 61)$ in recurrent miscarriages. The higher incidence of the chromosomal abnormalities in the first miscarriages than in recurrent ones were interpreted as that recurrent miscarriages were less likely caused by genetic factors. In our study, chromosomal analysis performed from the abortion materials of patients with RPL detected a major chromosomal abnormality in $31.9 \%$ of the cases, which is in accordance with the literature. Obtaining such a result form the abortion material can be helpful in the genetic counseling of the patient and couples will be informed about the recurrence risk of the detected genetic abnormality in the subsequent pregnancies. The result can narrow the list of workup needed for the patient.

In our study, cell proliferation did not occur in the culture of abortion materials of 115 cases $(46 \%)$ and our culture success rate was $54 \%$. The culture success rates reported in the literature before are very much higher than that of us. For example Be et al. reported a culture success rate of $95.1 \%{ }^{19}$, and Hogge et al. reported a rate of $94.6 \% .{ }^{20}$ Our lower culture success rate may be due to possible contamination of the samples during collection. Sterile collection of the abortion material is important as mentioned firstly by Stephenson et al., they mentioned that a higher culture success rate can be obtained by obtaining the material with dilatation and curettage rather than collection of spontaneously expelled tissue. ${ }^{21}$

Small structural chromosomal abnormalities like short tandem repeats, single nucleotide polymorphisms and copy number variants are known as polymorphisms. These generally have no clinical significance and are known to be responsible from most of the genetic variations in populations. Recent studies have mentioned the possible association of polymorphisms with reproductive failure and recurrent spontaneous miscarriages. ${ }^{22}$ In our study, we detected polymorphisms in $31.9 \%$ of the subjects and in $3.7 \%$ of the abortion samples. The most commonly detected polymorphism in our subjects was an increase in the centromeric heterochromatin region of the $1^{\text {st }}$ chromosome.

Thrombophilia disorders can lead to disordered placental perfusion because of thrombosis in the spiral arteries and intervillous space which can lead to problems of late pregnancy like 
late fetal loss and preeclampsia. But the association of hereditary thrombophilia's with early pregnancy loss is not clarified yet. The roles of hereditary thrombophilia's in RPL's have been extensively studied and a large amount of contradictory literature about this issue has accumulated. For example, Karata et al. recently compared the prevalence of factor $\mathrm{V}$ G1691A Leiden, Prothrombin G20210A, and MTHFR C677T mutations in patients with recurrent pregnancy losses (RPL) with that of the control group. ${ }^{7}$ They found that a significant difference existed only for the homozygous and heterozygous mutations of MTHFR C677T mutations, which seemed to have an association with RPL. Neither homozygous nor heterozygous mutations of Prothrombin G20210A and factor V G1691A were different between the groups. Another similar study of Habibovic et al. found that the frequency of factor V Leiden, Prothrombin G20210A but also MTHFR C677T mutations were similar in both the study and control group. ${ }^{8}$ In our study, among 495 women tested for polymorphisms in these genes, an abnormality was detected in 81 cases, the most frequent of which was MTHFR heterozygote mutation. The frequency of MTHFR heterozygote mutation in the normal control subjects was found to be $38.1 \%$. For example, Karata et al. ${ }^{7}$ recently compared the prevalence of factor V G1691A and MTHFR C677T mutations in patients with RPL with that of the of $11.1 \%(55 / 495)$ among our subjects.

\section{Conclusion}

RPL is a condition which has both psychological and economical adverse effects both for the couples and experts dealing with these patients. Highlighting the real cause will be beneficial for both. Detection of an underlying clinically insignificant thrombophilic disorder may not be so beneficial for the time being, since the benefits of anticoagulant treatment in terms of pregnancy outcome and the safety of such a treatment for the patient has not been proved yet. ${ }^{11,23,24}$ The most important issue with hereditary thrombophilia's is the prevention of maternal thrombosis and indication for anticoagulant treatments should be made according to the expected risk of thrombosis associated with the specific disorder. But, finding a cytogenetic abnormality in an aborted fetus or in any of the parents may decrease the further investigations that the couples have to undergo. This will be both time and cost saving in evaluation process of these patients.

\section{References}

1. Nybo Andersen AM, Wohlfahrt J, Christens P, Olsen J, Melbye M. Maternal age and fetal loss: population based register linkage study. BMJ 2000; 320: 1708-12.

2. Wilcox AJ, Weinberg CR, O'Connor JF, Baird DD, Schlatterer JP, Canfield RE, et al. Incidence of early loss of pregnancy. $N$ Engl J Med 1988;319: 189-94.

3. Practice Committee of the American Society for Reproductive Medicine. Definitions of infertility and recurrent pregnancy loss. Fertil. Steril 2008; 89: 1603.

4. Fawzy M, Shokeir T, El-Tatongy M, Warda O, El-Refaiey AA, Mosbah A. Treatment options and pregnancy outcome in women with idiopathic recurrent miscarriage: a randomized placebo-controlled study. Arch Gynecol Obstet 2008; 278: 33-8.

5. Sierra S, Stephenson M. Genetics of recurrent pregnancy loss. Semin Reprod Med 2006; 24: 17 24.

6. Toncheva D. Fragile sites and spontaneous abortions. Genet Couns. 1991; 2: 205-10.

7. Karata S, Aydin Y, Ocer F, Buyru A, Balci H. Hereditary thrombophilia, anti-beta2 glycoprotein $1 \mathrm{IgM}$, and anti-annexin $\mathrm{V}$ antibodies in recurrent pregnancy loss. $A m \mathrm{~J}$ Reprod Immunol 2012; 67: 251-5.

8. Habibovic Z, Zeybek B, Sanhal C, Eroglu Z, Karaca E, Ulukus M. Effects of inherited thrombophilia in women with recurrent pregnancy loss. Clin Exp Obstet Gynecol 2011; 38: 347-50.

9. Rey E, Kahn SR, David M, Shrier I. Thrombophilic disorders and fetal loss: a metaanalysis. Lancet 2003; 361: 901.

10. Dizon-Townson D, Miller C, Sibai B, Spong CY, Thom E, Wendel G Jr, et al. National Institute of Child Health and Human Development Maternal-Fetal Medicine Units Network. The relationship of the factor $\mathrm{V}$ Leiden mutation and pregnancy outcomes for mother and fetus. Obstet Gynecol 2005; 106: 517.

11. Practice bulletin no. 113: inherited thrombophilias in pregnancy. Obstet Gynecol 2010 ;116: 212-22

12. Elghezal H, Hidar S, Mougou S, Khairi H, Saad A. Prevalence of chromosomal abnormalities in couples with recurrent miscarriage. Fertil. Steril 2007; 88: 721-3. 
13. Peter S. Uzelac, MD, \& Sara H. Garmel, MD. Early Pregnancy Risks. In: Alan H. DeCherney, Lauren Nathan, editors. Current Diagnosis \& Treatment Obstetrics \& Gynecology, 10e.

14. Fischer J, Colls P, Escudero T, Munné S. Preimplantation genetic diagnosis (PGD) improves pregnancy outcome for translocation carriers with a history of recurrent losses. Fertil Steril 2010; 94: 283-9.

15. Franssen MT, Musters AM, van der Veen F, Repping S, Leschot NJ, Bossuyt PM, et al. Reproductive outcome after PGD in couples with recurrent miscarriage carrying a structural chromosome abnormality: a systematic review. Hum Reprod Update 2011; 17: 467-75.

16. Wells D. Advances in preimplantation genetic diagnosis. Eur J Obstet Gynecol Reprod Biol 2004; 115: 97-101.

17. Zhang HK, Luo FW, Geng Q, Li J, Liu QZ, Chen WB, et al. Analysis of fetal chromosomal karyotype and etiology in 252 cases of early spontaneous abortion. Zhonghua Yi Xue Yi Chuan Xue Za Zbi. 2011; 28: 575-8.

18. Kwinecka-Dmitriew B, Zakrzewska M, LatosBieleñska A, Skrzypczak J. Frequency of chromosomal aberrations in material from abortions. Ginekol Pol 2010; 81: 896-901.

19. Be C, Velásquez P, Youlton R. Spontaneous abortion: cytogenetic study of 609 cases. Rev Med Chil 1997; 125: 317-22.
20. Hogge WA, Byrnes AL, Lanasa MC, Surti U. The clinical use of karyotyping spontaneous abortions. Am J Obstet Gynecol 2003; 189: $397-$ 400.

21. Stephenson MD, Awartani KA, Robinson WP. Cytogenetic analysis of miscarriages from couples with recurrent miscarriage: a casecontrol study. Hum Reprod 2002; 17: 446-51.

22. Madon PF, Athalye AS, Parikh FR. Polymorphic variants on chromosomes probably play a significant role in infertility. Reprod. Biomed. Online 2005; 11: 726-32.

23. Kaandorp S, Di Nisio M, Goddijn M, Middeldorp S. Aspirin or anticoagulants for treating recurrent miscarriage in women without antiphospholipid syndrome. Cochrane Database Syst Rev 2009; 21:CD004734.

24. Visser J, Ulander VM, Helmerhorst FM, Lampinen K, Morin-Papunen L, Bloemenkamp KW, et al. Thromboprophylaxis for recurrent miscarriage in women with or without thrombophilia. HABENOX: a randomised multicentre trial. Thromb Haemost 2011; 105: 295301. 